\title{
Essais de traitement du parasitisme gastro-intestinal du dromadaire au moyen du Tétramisole Premières observations
}

\author{
par M. GRABER \\ Institut d'Elevage et de Médecıne Vétérınarre des pays tropicaux \\ Laboratoire de Farcha, Fort-Lamy
}

\begin{abstract}
RÉSUMÉ
L'cưeur estime que le Némıcıde admınistré par voie parentérale à la dose de $10 \mathrm{mg} / \mathrm{kg}$ est susceptible de délruire la quası-total té des Oesophagostomum coilumbianum, Haemoncus longistipes et Impalasa nudicollis présents dans la caıllette et l'intesın du dromadare tchadıen, Sur Strongylordes popillosus, les résultats semblent moins satisfaisants.

La voie buccale est inutilisable car les doses thérapeutiques et les doses létales se chevauchent étroltement.

Le médicament demande à être manipulé avec précaution car, déjà vers $12-15 \mathrm{mg} / \mathrm{kg}$ en sous-cutanée, des manifestations d'intolérance pius ou mains spectaculaıres se font jour.
\end{abstract}

\section{INTRODUCTION}

Une série d'enquêtes effectuées au Tchad de 1954 à 1967 (GRABER et Coll., 1967 ; Anonyme, 1967) sur plus de 150 dromadaires a montré que le parasitisme intestinal dominant était à base de Nématodes. Hult espèces au moins ont été recueıllies. Les plus dangereuses sont Hoemoncus longistipes (RAILLIET et HENRY, 1909), Strongyloides papillosus (WEDL, 1856), Impalaia nudicollis (NÖNNING, 1931) ef Buckleyuris globuloso (Von LINSTOV, 1901). La mortalité dans le Nord-Est du pays est estımée à environ 4 p. 100 chaque année.

Devant cette situation, des essais de tratement ont été tentés au moyen de la Phénothiazine et du Thiabendazole (GRABER, 1966 a). Le premier médicament est trop toxique et le second trop onéreux, car il faut des doses élevées de l'ordre de $300 \mathrm{mg} / \mathrm{kg}$.

Aussi, à la demande de la Société Parisienne d'expansion chimıque *, une expérience a-t-elle été réalısée en utılısant le Dı-tétrahydro-2,5,6, Phényl-6 Imıdazo (2-1) b thiazole, chlorhydrate ou Tétramisole ** dont l'action sur les Nématodes du tractus digestif du zébu est par allleurs bien connue (GRABER, 1966 b).

Les premiers résultats obtenus permettent, dès à présent, de se faire une idée des conditions et des possibılıtés d'emploi du médicament dans l'espèce camelıne.

* Qui a financé ces essars.

** Némicide Spécia. 


\section{MATÉRIEL ET MÉTHODE}

\section{A. - Les animaux d'expérience.}

18 dromadaires pesant de 250 à $350 \mathrm{~kg}$ ont été achetés dans les préfectures du Batha (7) et du Bahr el Ghazal (11) en juin et en octobre 1967. Il s'agissait en majorité d'animaux de bât âgés : 4 jeunes d'élevage seulement, diversement tarés, ont été vendus.

Ils ont été répartis de la façon suivante :

- essais thérapeutiques proprement dits : 15 ,

一 témoins : 3.

Dans l'ensemble, leur état d'entretien étaif médıocre ef ıls hébergeaient un grand nombre d'Helminthes (tableau 1).

Dans 80 p. 100 des cas, les parasites étalent associés entre eux. L'association la plus fréquente comprenait 5 espèces: $S$. papillosus, $O$. columbianum, $H$. longistipes, I. nudicollis et B. globulosa.

Les essais ont eu lieu de juin à novembre à une époque où l'infestation par les Nématodes de la caillette et de l'intestın est importante (GRABER, 1967).

\section{B. - Méthode.}

Elle a été décrite dans un précédent travail (GRABER, 1966). Nous n'y reviendrons done point.
L'anthelminthique a été administré :

- par voie buccale,

- par voie sous-cutanée (solutions à 10 et à 7,5 p. 100) *. Vu le volume des doses à injecter, le médicament a été introduit de chaque côté du corps en arrière de l'épaule. Au bout d'un mois, il n'existait ni abcès, ni nodules au point d'injection.

Les animaux ont été traités directement, sans mise à la diète préalable.

\section{RÉSULTATS}

10 Témoins (tableau no 2 ).

$2^{\circ}$ Action sur les Trématodes.

Le Tétramisole, quelle que soit la dose, est sans action sur Shistosoma bovis des veines mésentérıques.

\section{$3^{\circ}$ Action sur les Cestodes.}

Le médicament est à peu près inefficace sur les grands Cestodes de l'intestin, sauf sur Moniezio benedeni qui, à $15 \mathrm{mg} / \mathrm{kg}$ sous la peau, est expulsé dans la proportion de 10 p. 100.

* Némicide Spécia.

TABLEAU No $\mathrm{I}$

Nombre d'animaux atteints et espèces parasites rencontrées

\begin{tabular}{|c|c|c|c|c|}
\hline \multirow{2}{*}{ Es pèces enc a us e } & \multicolumn{2}{|c|}{ Moussoro } & \multirow{2}{*}{$\frac{\mathrm{B} a \mathrm{tha}}{\text { trattês }}$} & \multirow{2}{*}{$T \circ t a 1$} \\
\hline & traitês & tếmoins & & \\
\hline Schistosoma bovis & 4 & - & -- & 4 \\
\hline Moniezia expansa & 2 & -- & 2 & 4 \\
\hline Moniezia benedeni & 2 & -- & -- & 2 \\
\hline Stilesia giobipunctata & 4 & 1 & 6 & 11 \\
\hline Avitellina woodlandi & -- & 1 & - & 1 \\
\hline Eohinococous polymorphus & 1 & 1 & 5 & 7 \\
\hline Oesophagostomum columbianum & 6 & 1 & 6 & 13 \\
\hline Strongyloides papillosus & B & - & 7 & 15 \\
\hline Haemonous tongistipes & 6 & 3 & 7 & 16 \\
\hline Impalaia nudicolits & 5 & 1 & 6 & 12 \\
\hline Buckleyuris globulosa & 4 & 2 & 3 & 9 \\
\hline
\end{tabular}




\begin{tabular}{|c|c|}
\hline He 1 m $1 \mathrm{nt}$ the $\mathrm{s}$ enc $\mathrm{c}$ use & $\begin{array}{l}\text { Poids (en g.) ou } \\
\text { Nombre de parasites (moyenne) }\end{array}$ \\
\hline Stizesia globipunctata & $0,5 \mathrm{~g}(1)^{+}$ \\
\hline Avitelina woodlandi & $11 \mathrm{~g}(1)$ \\
\hline Echinocoocus poiymorphus & $4 \quad g(2)$ \\
\hline Oesophagostomum columbianum & $2 g(1)$ \\
\hline Haemonous Zcngistipes & $1,250(3)$ \\
\hline Impalaia nudieclitis & (1) \\
\hline Buckleyuris alobulosa & $12\langle 2\rangle$ \\
\hline
\end{tabular}

+ = Nombre d'animaux parasités.

40 Action sur les Nématodes.

4. 1. Examens coproscopiques (tableau $n^{\circ} 3$ ).

4. 2. Action du Téframisole sur strongyloïdes papillosus de l'infesfin (tableau $n^{\circ} 4$ ).

4. 3. Action du Tétramisole sur oesophagostomum columbianum du cacum (tableauno 5 ).

4. 4. Action du Tétramisole sur Haemoncus longistipes de la caillette ef Impaloio nudicollis de l'infestin (tableau $n^{0} 6$ ).

4. 5. Action du Téframisole du Buckleyuris globulosa du cacum (tableau no 7).

4. 6. Discussion.
- Par la vole buccale, si le Tétramisole commence à donner de bons résultats sur Impalara nudicollis et Oesophagostomum columbionum à partir de $30 \mathrm{mg} / \mathrm{kg}$, il faut au moins $40 \mathrm{mg} / \mathrm{kg}$ pour obtenır un effet satısfaisant sur Haemoncus longistipes. Quant à Strongyloides popillosus, il n'est pas sûr qu'à cette dose, tous les parasites disparaissent.

- Par la voie parentérale, le médicament, à partir de $10 \mathrm{mg} / \mathrm{kg}$, semble se montrer très actif à l'égard d'Haemoncus longistipes, d'impalaia nudicoliss, et d'Oesophagostomum columbianum. Strongyloides papillosus est beaucoup plus

TABLEAU N $N^{\circ} I I I$

Nombre d'oeufs au gramme de matière fécale (moyenne)

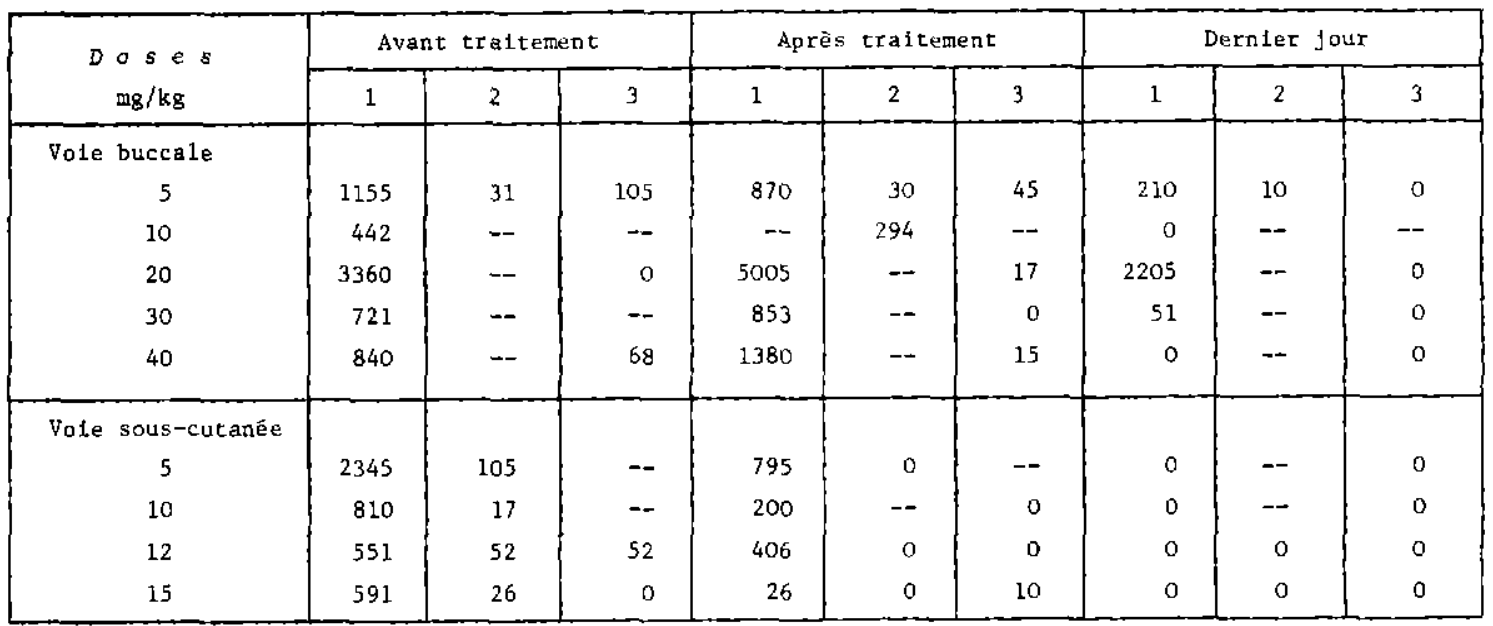

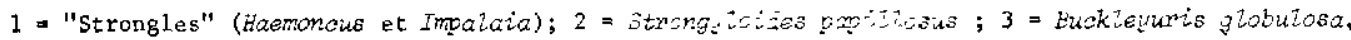


TABLEAU $N^{\circ}$ IV

Actlon du tëtramisole sur Strongylotdes popizlosus adulte.

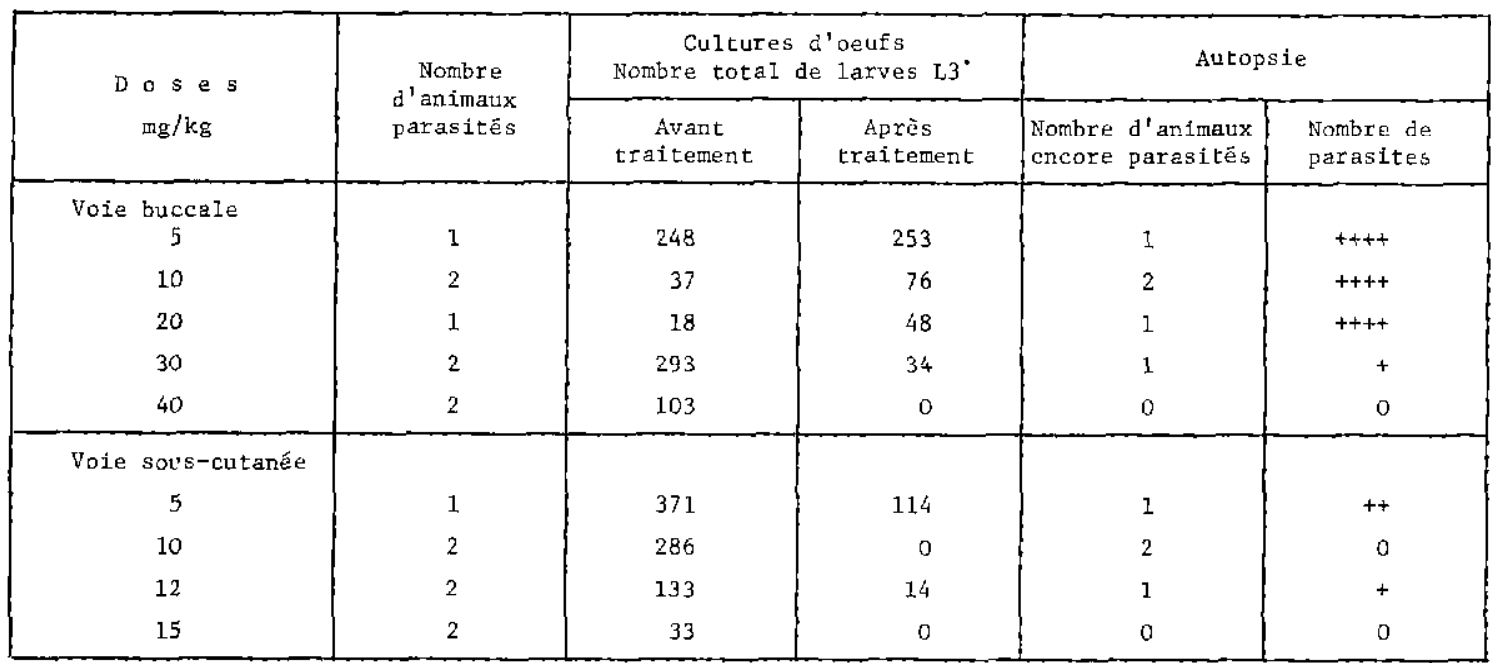

- a dans une goutte de suspension aqueuse provenant des boites de Petri ayant servi aux coprocultures

$+=$ moins de $10 ;++=$ de 10 à 50 ; $++++=50$ et au-delà.

TABLEAH $\mathrm{N}^{\circ} \mathrm{V}$

Action du têtramisole sur oescohagostomum columbianum

\begin{tabular}{|c|c|c|c|c|c|}
\hline $\begin{array}{l}\text { Doses } \\
\mathrm{mg} / \mathrm{kg}\end{array}$ & $\begin{array}{l}\text { Nombre } \\
\text { d'animaux } \\
\text { parasités }\end{array}$ & $\begin{array}{c}\text { Nombre } \\
\text { d'oesophagostomes } \\
\text { expu1sés }\end{array}$ & $\begin{array}{l}\text { Présence ou } \\
\text { absence (-) } \\
\text { de parasites } \\
\text { a } 1 \text { 'autopie }\end{array}$ & $\begin{array}{c}\text { Nambre } \\
\text { d'animaux } \\
\text { totalement } \\
\text { déparasités }\end{array}$ & Efficacitë \\
\hline \multicolumn{6}{|c|}{ Voie buccale } \\
\hline 5 & 1 & 1 & -- & 1 & -- \\
\hline 10 & 2 & 22 & 38 & 0 & Faible \\
\hline 20 & 1 & 1 & 44 & 0 & nulle \\
\hline э० & 1 & 8 & - & 1 & bonne \\
\hline 40 & $1^{+}$ & -- & -- & 1 & bonne \\
\hline \multicolumn{6}{|c|}{ Voie sous-cutanée } \\
\hline 5 & 1 & o & 37 & 1 & nulle \\
\hline 10 & 2 & 4 & -- & 2 & bonne \\
\hline 12 & 1 & 4 & -- & 1 & bonne \\
\hline 15 & 2 & 8 & - & 2 & bonne \\
\hline
\end{tabular}

$+=\operatorname{sur}$ culture d'oeufs.

résistant et à $12 \mathrm{mg} / \mathrm{kg}$ on retrouve quelques individus - en très petı nombre. Buckleyuris globulosa est expulsé vers $12 \mathrm{mg} / \mathrm{kg}$.

L'anthelminthique est inférieur au Thiaben- dazole lorsqu'il s'agit de Strongyloides papillosus. Par contre, sur Buckleyuris globulosa, les résultats semblent satisfaisants, et le Némicide peut être préconisé toutes les fois que la Trichurose camelıne sévit massivement dans un effectif. 
TABLEAL $\mathrm{N}^{\circ} \mathrm{VI}$

Action du tétramisole sur F́aemoncus iorgzstiges et Impaiaia nudicoilis.

\begin{tabular}{|c|c|c|c|c|c|c|c|c|c|c|c|}
\hline \multirow{3}{*}{$\begin{array}{l}\text { Doses } \\
\mathrm{mg} / \mathrm{kg}\end{array}$} & \multicolumn{5}{|c|}{ Cu1ture d'oeufs ${ }^{+}$} & \multirow{2}{*}{\multicolumn{2}{|c|}{$\begin{array}{l}\text { Présence ou } \\
\text { absence de } \\
\text { parasites à } \\
\text { 1'autopsie }\end{array}$}} & \multirow{2}{*}{\multicolumn{2}{|c|}{$\begin{array}{l}\text { Nombre d'animaux } \\
\text { totalement déparasités }\end{array}$}} & \multirow{2}{*}{\multicolumn{2}{|c|}{ Efficacitē }} \\
\hline & \multicolumn{3}{|c|}{$\begin{array}{c}\text { Avant } \\
\text { traitement }\end{array}$} & \multicolumn{2}{|c|}{$\begin{array}{c}\text { Après } \\
\text { traitement }\end{array}$} & & & & & & \\
\hline & I & $\mathrm{H}$ & $\mathrm{I}+\mathrm{H}$ & I & $\mathrm{H}$ & I & $\mathrm{H}$ & I & $\mathrm{H}$ & I & $\mathrm{H}$ \\
\hline \multicolumn{2}{|c|}{ Vole buccale } & & & & & & & & & & \\
\hline 5 & 23 & 2 & 25 & 0 & 0 & ++ & 160 & 0 sur 1 & 0 sur 1 & faible & nulle \\
\hline 10 & & & 11 & \multicolumn{2}{|c|}{0} & ++ & 3 & 1 sur 2 & 1 sur 2 & moyenne & Eatble \\
\hline 20 & - & 40 & 40 & - & 0 & -- & 534 & -- & 0 sur 1 & -- & nulle \\
\hline 30 & 6 & -- & 6 & 0 & -- & 0 & - & 2 sut 2 & -- & bonne & -- \\
\hline 40 & & & 7 & \multicolumn{2}{|c|}{0} & 0 & 0 & 2 sur 2 & 2 sur 2 & bonne & bonne \\
\hline \multicolumn{3}{|c|}{ Vole sous-cutanée } & & & & & & & & & \\
\hline 5 & 25 & 27 & 52 & 0 & 0 & 0 & 35 & 1 sur 1 & 0 sur 1 & bonne & faible \\
\hline 10 & 2 & 2 & 4 & 0 & 0 & 0 & 0 & 2 sur 2 & 2 sur 2 & bonne & bonne \\
\hline 12 & -- & 5 & 5 & -- & 0 & -- & 0 & -- & 2 sur 2 & bonne & bonne \\
\hline 15 & & & 8 & & & 0 & 0 & 1 sur 1 & $2 \operatorname{sur} 2$ & bonne & bonne \\
\hline
\end{tabular}

+ dans une goutte de suspension aqueuse provenant des boites de Petri ayant serv1 aux coprocultures.

I = Impalaia nudicollis ; $\mathrm{H}=$ Haemonous longistipes.

TABLEAU $\mathrm{N}^{\circ}$ VII

Action du tétramisole sur Bucklezuris globulosa.

\begin{tabular}{|c|c|c|c|c|c|}
\hline $\begin{array}{l}\text { Doses } \\
\text { mg } / \mathrm{kg}\end{array}$ & $\begin{array}{l}\text { Nombre } \\
\text { d'animaux } \\
\text { parasitês }\end{array}$ & $\begin{array}{l}\text { Nombre de } \\
\text { parasites } \\
\text { expulsês }\end{array}$ & $\begin{array}{c}\text { Nombre de } \\
\text { parasites } \\
\text { restant ã l'autopsie }\end{array}$ & $\begin{array}{c}\text { Animaux } \\
\text { déparasitês }\end{array}$ & Efficacité \\
\hline \multicolumn{6}{|l|}{ Vole buccale } \\
\hline 5 & 1 & 3 & 27 & 0 sur 1 & faible \\
\hline 10 & 1 & 0 & 18 & 0 sur 1 & nulle \\
\hline 20 & 1 & 3 & 35 & 0 sur 1 & faible \\
\hline 40 & 1 & 55 & 0 & 1 sur 1 & bonne \\
\hline \multicolumn{6}{|c|}{ Voie sous-cutanée } \\
\hline 12 & 2 & 6 & 0 & $2 \operatorname{sur} 2$ & bonne \\
\hline 15 & 1 & 5 & 0 & 1 sur 1 & bonne \\
\hline
\end{tabular}

\section{ACTIVITÉ DU TÉTRAMISOLE}

II agit très rapidement sur les Nématodes qui sont élımınés en totalité au bout de 72 heures, l'évacuation maximaie ayant lieu 48 heures après le traitement.

Seuls, apparaissent les parasites du cæcum et du gros intestin, c'est-d̀-dire Buckleyuris globulosa et Oesophagostomum columibianum.

Strongylordes papiliosus, Impalaia nudicollis et
Haemoncus longistipes sont détruits dans le tractus digestif et leur présence ne peut être révélée que par des examens coproscopiques ou des cultures d'œufs.

En coproculture, les cufs de Nématodes donnent encore des larves infestantes $L 3$ au bout de 60 heures.

Compte tenu des délars d'expulsion et de la résistance des œufs à l'anthelminthique, les chameaux traités ne sẹront mis sur des pâturages 
neufs sans parasites que 4 jours après la fin du traltement.

\section{TOXICITÉ}

Les résultats figurent au tableau no 8 .

Les signes de l'ıntoxication par le Némicide chez le dromadare varient selon le mode d'administration.

\section{Par voie buccale.}

IIs sont manifestes à partir de $30 \mathrm{mg} / \mathrm{kg}$. $11 \mathrm{~s}$ se traduisent par un état dépressif général, avec des coliques sourdes, peu violentes qui débutent un quart d'heure après l'absorption du médicament.

II n'y a pas de phase d'excitabilité. L'animal se couche, cesse de ruminer et de manger. On observe un peu de larmoiement et quelques rares émıssions d'urine ou de crottes, mais sans diarrhée.

L'animal ne réagit pas quand on cherche à le remettre debout.

Cet état, loin de cesser, se prolonge au cours des jours suivants.

Les conséquences ne tardent pas à se faire sentir sous la forme d'une perte de poids brutale qui peut atteindre $15 \mathrm{~kg}$ en 3 jours.

Le chameau meurt dans le marasme le plus complet en un laps de temps qui va de 4 à 9 jours.

\section{Par voie sous-cutanée.}

A $15 \mathrm{mg} / \mathrm{kg}$, environ 12 minutes après le traitement, on note :

- de l'inquiétude,

- de l'excitation, le chameau cherchant à fuir, malgré les obstacles placés sur son chemın. Cette période est brève. Elle est suivie immédıatement de violentes colıques : l'animal est couvert de sueur; il se lève, se couche en décubitus latéral, les membres en extension et la tête allongée. Il urine abondamment et les crottes, à l'état liquıde, fusent à plusıeurs mètres en arrière.

Ces coliques durent plus de 3 heures. Elles s'accompagnent de mouvements, de mastication, de larmolement, d'une agitation frénétique de la lèvre inférieure, de frissons et de contractions des muscles de l'épaule. La respiration s'accélère et des gouttes de sueur tombent sur le sol en pluie.

Lorsque ces phénomènes cessent, le sujet est dans un état lamentable, d̀ tel point que l'un des deux dromadaires traités mettra un mols à retrouver son aspect normal.

Le second, plus âgé, a beaucoup mieux supporté l'anthelminthique.

A $12 \mathrm{mg} / \mathrm{kg}$, les réactions sont moins violentes.

A $10 \mathrm{mg} / \mathrm{kg}$, elles sont encore plus discrètes : inquiétudes, relâchement des sphincters anaux, arrêt de la rumination durant 20 minutes.

TABLEAL $N^{\circ}$ VIII

Tox $1 \subset$ c it

\begin{tabular}{|c|c|c|c|}
\hline $\begin{array}{l}\text { Doses } \\
\mathrm{mg} / \mathrm{kg}\end{array}$ & $\begin{array}{c}\text { Nombre } \\
\text { d'animaux } \\
\text { utillsês }\end{array}$ & Mortalité & observations \\
\hline \multicolumn{4}{|l|}{ Voie buccale } \\
\hline 5 & 1 & 0 & --- \\
\hline 10 & 2 & 0 & -- \\
\hline 20 & 1 & 0 & --- \\
\hline 30 & 2 & 2 & 7 et $4 \mathrm{j}$. après traitement. \\
\hline 40 & 2 & 2 & 4 et $9 \mathrm{j}$. après trałtement. \\
\hline \multicolumn{4}{|c|}{ Vole sous-cutanée } \\
\hline 5 & 1 & 0 & -- \\
\hline 10 & 2 & 0 & $-\cdots$ \\
\hline 12 & 2 & 0 & $--\cdots$ \\
\hline 15 & 2 & 0 & $\begin{array}{l}\text { L'un des animaux n'a retrouvé } \\
\text { son poids normal qu'aul bout } \\
\text { de } 1 \text { mois. }\end{array}$ \\
\hline
\end{tabular}


30 Pratiquement, le Tétramisole ne peut être administré que par voie sous-cutanée car, par voie buccale, les doses thérapeutiques sont le plus souvent mortelles ( $30 \mathrm{mg} / \mathrm{kg}$ ).

La dose de $10 \mathrm{mg} / \mathrm{kg}$ paraît devoir être retenue à condition de prendre des précautions dont la principale est de connaître exactement le poids de l'animal. Une erreur de quelques dizaınes de kilogrammes risque d'entraîner des conséquences fâcheuses.

La toxicité relative du Némicide semble être un obstacle à son emploi systématique dans le traitement des strongyloses gastro-intestinales du chameau.

Seuls des essais sur une plus grande échelle et dans d'autres conditions permettront de tirer des conclusions définitives.

\section{PRISE DE POIDS}

Les chameaux laissés en étable et traités à 12 ef $15 \mathrm{mg} / \mathrm{kg}$ par voie sous-cutanée ont été régulièrement pesés du 27.10 .67 au 21.11 .67 pour les premiers et du 10.10.67 au 4.11.67 pour les seconds (tableau no 9 ).

\section{CONCLUSIONS}

Lors d'essais lımités effectués en 1967 sur 18 dromadaires originaires du centre et de I'Ouest du Tchad, il a été constaté que :

$1^{\circ}$ Le Némicide est totalement inactif sur des Trématodes, tels que Shistosomo bovis.

$2^{\circ} \mathrm{Il}$ en est de même pour les Anoplocephalidae de l'intestin, agents du Ténıasis. Seules, des doses très élevées permettent d'assurer l'élimination de moins de 10 p. 100 des Cestodes présents (Moniezia benedeni).

30 Sur les Nématodes.

- Par voie buccale, la destruction des associations à base de Strongyloides papillosus, Oesophogostomum columbianum, Haemoncus longistipes, Impolara nudicallis et Buckleyuris globulosa est possible à partir de $40 \mathrm{mg} / \mathrm{kg}$.

- Par la voie sous-cutanée, le même effet (souf sur Strongyloides papillosus) est obtenu vers $10 \mathrm{mg} / \mathrm{kg}$.

40 Malheureusement, le Tétramisole per os tue tous les animaux d̀ 30 et $40 \mathrm{mg} / \mathrm{kg}$. Ce mode d'administration est donc à rejeter.

Par voie parentérale, la tolérance est meilleure, mais déjà vers $12-15 \mathrm{mg} / \mathrm{kg}$, l'injection est suivie de manifestations violentes, souvent spectaculare.

Dans ces conditions, le Némicide, à la dose thérapeutique, demande à être manipulé avec prudence et ne sera utilisé que dans la mesure où l'on peut connaître exactement le poids du chameau.

$5^{\circ}$ En étable, à $12 \mathrm{mg} / \mathrm{kg}$ l'augmentation de poids est de 7,6 p. 100 en 1 mols.

\section{REMERCIEMENTS}

L'auteur tient à remercier vivement la Société Parisienne d'Expansion Chimique pour sa contributıon appréciable à la réalısation de ces essais et en portıculier à Monsieur le Docteur A. FERRIOT.

TABLEAU $\mathrm{X}^{\circ} \mathrm{IX}$

Prise de polds

\begin{tabular}{|c|c|c|c|}
\hline \multicolumn{4}{|c|}{$\left.1^{\circ}\right) 12 \mathrm{mg} / \mathrm{kg}-$ Poids total en $\mathrm{kg}$ (deux anfmaux) } \\
\hline 27.10 .67 & \multicolumn{2}{|l|}{4.11 .67} & 22.11 .67 \\
\hline 514,6 & \multicolumn{2}{|l|}{559,9} & 554 \\
\hline Pourcentage d'augmentation & \multicolumn{2}{|c|}{$+8.89 .100$} & $+7,6 \mathrm{p} .100$ \\
\hline \multicolumn{4}{|c|}{ 2*) $15 \mathrm{mg} / \mathrm{kg}$ - Poids total en $\mathrm{kg}^{+}$(deux animaux) } \\
\hline $12 \cdot 10.67$ & 19.10 .67 & 27.10 .67 & 4.11 .67 \\
\hline $\begin{array}{l}639,2 \\
\text { Pourcentage d'augmentation }\end{array}$ & $\begin{array}{l}647,7 \\
+1,3 \text { p. } 100\end{array}$ & $\begin{array}{l}641,6 \\
+0,3 \text { p. } 100\end{array}$ & $\begin{array}{l}653,5 \\
+\quad 2,2 p .100\end{array}$ \\
\hline
\end{tabular}

+ L'un des dromadalres a mal supporté l'injection de Némicide et a mis près d'un mols à retrouver son poíds de départ. 


\section{SUMMARY}

Experiments of gastro-intestinal parasifism treatment of dromedary with Tetramisole. First observations

The Nemicide admınıstraled by parental way at the dose level of $10 \mathrm{mg} / \mathrm{kg}$ is estimated by the author liable to destroy nearly the whole of Oesophogostonum columbionum, Haemoncus longistipes and Impalaro nudicollis present in the abomasum and the intestınes of dromedary from Chad. The results are less good on Strongyloides papillosus.

The buccal way is not utilizable because the therapeutic and lethal dosis are very neor.

The medicament must be utılizable with precaution because more or less spectacular manifestations of intolerance appear from $12-15 \mathrm{mg} / \mathrm{kg}$ subcutaneous injections.

\section{RESUMEN}

Ensayos de tratamiento del parasitismo gastro-intestinal del dromedario mediante el tetramisolo. Primeras observaciones

Según el autor, el nemicido, administrodo por via parenteral en dosis de $10 \mathrm{mg} / \mathrm{kg}$ puede destruir casi la totalidad de Oesophagostomum columbionum, Haemoncus longıstipes e impoloia nudicoliss encontrados en el cuajar y el estómago del dromedario de Chad. En lo concerniente Strongyloides papiliosus, los resultados parecen menos salisfactorios.

Na se puede utilizar la via oral porque las dosis terapeuticas y letales son muy proximas.

Se necesita manipular el dicho medicamento con precaución porque, ya con una inyección subcutánea de unos $12-15 \mathrm{mg} / \mathrm{kg}$, ocurren manifestaciones de intolerancia.

\section{BIBLIOGRAPHIE}

1. GRABER (M.). - Etude dans certaines conditions africaines de l'action antiparasitaire du Thiabendazole sur divers Helminthes des animaux domestiques. II. Dromadaire. Rev. Elev. Méd. Vét. Pays Trop., 1966, 19, 4, 527-43 (a).

2. GRABER (M.). - Etude du pouvoir anthelmintique du Tétramisole (16.535 R. P.) sur divers Helminthes du zébu de la République du Tchad. Rev. Elev. Méd. Vét. Pays Trop., 1966, $19,4,511-26$ (b).

3. GRABER (M.). - Etude préliminaire de la biologie d'Haemoncus longistipes (Railliet ef
Henry, 1909) du dromadaire (Camelus dromedarius). Résultats obtenus au Laboratoire. Rev. Elev. Méd. Vét. Pays Trop., 1967, 20, 2, 213-25.

4. GRABER (M.), TABO (R.) et SERVICE (J.). Enquêtes sur les Helminthes du dromadaire tchadien. Etude des strongyloses gastro-intestinales ef de l'Haemoncuse à Haemoncus longistipes. Rev. Elev. Méd. Vét. Pays Trop., 1967, 20, 2, 227-54.

5. ANONYME. - Helminthes ef Helminthiases. Bilan d'activité. Rap. Ann. Lab. Farcha, 1967. t. III, 38-40. 